OPEN ACCESS

Edited by:

Yubing $\mathrm{Si}$,

Zhengzhou University, China

Reviewed by:

Qinghua Liao,

University of Barcelona, Spain

Yuan Zhao,

Henan University, China

*Correspondence:

Pengfei $\mathrm{Li}$

pli4@/uc.edu

Specialty section: This article was submitted to

Theoretical and Computational Chemistry,

a section of the journal

Frontiers in Chemistry

Received: 07 June 2021

Accepted: 01 July 2021

Published: 22 July 2021

Citation:

Li P (2021) Bridging the 12-6-4 Model and the Fluctuating Charge Model.

Front. Chem. 9:721960.

doi: $10.3389 /$ fchem.2021.721960

\section{Bridging the 12-6-4 Model and the Fluctuating Charge Model}

\author{
Pengfei $L i{ }^{*}$ \\ Department of Chemistry and Biochemistry, Loyola University Chicago, Chicago, IL, United States
}

Metal ions play important roles in various biological systems. Molecular dynamics (MD) using classical force field has become a popular research tool to study biological systems at the atomic level. However, meaningful MD simulations require reliable models and parameters. Previously we showed that the 12-6 Lennard-Jones nonbonded model for ions could not reproduce the experimental hydration free energy (HFE) and ion-oxygen distance $(I O D)$ values simultaneously when ion has a charge of +2 or higher. We discussed that this deficiency arises from the overlook of the ion-induced dipole interaction in the 12-6 model, and this term is proportional to $1 / r^{4}$ based on theory. Hence, we developed the 126-4 model and showed it could solve this deficiency in a physically meaningful way. However, our previous research also found that the 12-6-4 model overestimated the coordination numbers (CNs) for some highly charged metal ions. And we attributed this artifact to that the current 12-6-4 scheme lacks a correction for the interactions among the first solvation shell water molecules. In the present study, we considered the ion-included dipole interaction by using the 12-6 model with adjusting the atomic charges of the first solvation shell water molecules. This strategy not only considers the ion-induced dipole interaction between ion and the first solvation shell water molecules but also well accounts for the increased repulsion among these water molecules compared to the bulk water molecules. We showed this strategy could well reproduce the experimental HFE and IOD values for $\mathrm{Mg}^{2+}, \mathrm{Zn}^{2+}, \mathrm{Al}^{3+}, \mathrm{Fe}^{3+}$, and $\mathrm{In}^{3+}$ and solve the $\mathrm{CN}$ overestimation issue of the 126-4 model for $\mathrm{Fe}^{3+}$ and $\mathrm{In}^{3+}$. Moreover, our simulation results showed good agreement with previous ab initio MD simulations. In addition, we derived the physical relationship between the $C_{4}$ parameter and induced dipole moment, which agreed well with our simulation results. Finally, we discussed the implications of the present work for simulating metalloproteins. Due to the fluctuating charge model uses a similar concept to the 12-6 model with adjusting atomic charges, we believe the present study builds a bridge between the 12-6-4 model and the fluctuating charge model.

Keywords: metal ion, force field, molecular dynamics, 12-6-4, fluctuating charge model, ion-induced dipole interaction, metalloproteins

\section{INTRODUCTION}

Metal ion plays significant roles in various biological processes (Thomson and Gray, 1998; Andreini et al., 2004; Woodson, 2005; Waldron and Robinson, 2009; Kepp, 2012). Molecular dynamics (MD) simulations has become an important tool for studying biomolecules (Duan and Kollman, 1998; Lindorff-Larsen et al., 2011; De Vivo et al., 2016; Hollingsworth and Dror, 2018). It can provide 
information with atomic details and high time resolution. Meaningful MD simulations require reliable models and parameters, but it is challenging to develop accurate models for metal ions (Li and Merz, 2017). Different force field models have been developed for simulating metal ions in biological systems. For example, the bonded model, (Peters et al., 2010), the cationic dummy atom model, (Duarte et al., 2014), the nonbonded model, (Li et al., 2013), and the polarizable models (Sakharov and Lim, 2009; Zhang et al., 2012). Among these models, the nonbonded model is one of the most widely used models because of its simplicity and transferability.

In the past decade, Li, Merz, and co-workers found that the widely used 12-6 Lennard-Jones nonbonded model could not reproduce the experimental hydration free energy (HFE) and ionoxygen distance (IOD) simultaneously when metal ion has a charge of +2 or higher (Li et al., 2013; Li and Merz, 2014; Li et al.,2015a; Li et al., 2015b). They attributed this deficiency to that the 12-6 nonbonded model did not consider the ion-induced dipole interaction. Because the ion-induced dipole interaction is proportional to $1 / r^{4}$, where $r$ is the atomic distance, they proposed the 12-6-4 model and parameterized it for various atomic ions ( $\mathrm{Li}$ and Merz, 2014; Li et al.,2015a; Li et al., 2015b). The 12-6-4 model could accurately reproduce the experimental HFE and IOD simultaneously after careful parameterization, which successfully solved the deficiency of the 12-6 model ( $\mathrm{Li}$ and Merz, 2014; Li et al., 2015a; Li et al., 2015b). Moreover, Merz and co-workers have showed that the 12-6-4 model could well simulate the chelate effect (Sengupta et al., 2018) and the thermodynamics of ion binding in a metalloprotein system (Song et al., 2020). However, it was found that for some highly charged metal ions such as $\mathrm{Fe}^{3+}$ and $\mathrm{In}^{3+}$, the coordination number $(\mathrm{CN})$ value was overestimated by the 12 6-4 model (Li et al., 2015a). For example, $\mathrm{Fe}^{3+}$ has an experimental $\mathrm{CN}$ of 6, (Marcus, 1988), but the 12-6-4 model predicted its $\mathrm{CN}$ value as 6.8-6.9 when using the TIP3P, SPC/E, or TIP4P $\mathrm{EW}_{\mathrm{EW}}$ water model (Li et al., 2015a). Recently, Li, Merz, and co-workers have parameterized the 12-6-4 for various ions in conjunction with four new water models (Li et al., 2020; Li et al., 2021; Sengupta et al., 2021). But this issue still exists: the 12-6-4 model predicted a $\mathrm{CN}$ of $6.4-6.7$ for $\mathrm{Fe}^{3+}$ when using the OPC3, OPC, TIP3P-FB, or TIP4P-FB water model (Li et al., 2021). It was proposed that this artifact was due to an inaccurate description of the water-water interactions inside the first solvation shell, which effect is not significant for monovalent and divalent metal ions but becomes more severe for the highly charged ions (Li et al., 2015a). To be more specific, a metal ion with a higher charge will cause larger induced dipoles of the first solvation water molecules, yielding a stronger repulsion between these water molecules. However, the current 12-6-4 model implementation does not take this effect into account, although it considers the ioninduced dipole interactions between the metal ion and surrounding water molecules.

In the present study, we tried to solve the $\mathrm{CN}$ overestimation issue by using a 12-6 model with increasing the dipole moments of the first solvation shell water molecules to account for the ioninduced dipole effect. Specifically, we studied the ion-aqueous systems containing $\mathrm{Mg}^{2+}, \mathrm{Zn}^{2+}, \mathrm{Al}^{3+}, \mathrm{Fe}^{3+}$, and $\mathrm{In}^{3+}$. We showed that this treatment could well reproduce the experimental HFEs and IODs for these ions, and solve the $\mathrm{CN}$ overestimation issue for $\mathrm{Fe}^{3+}$ and $\mathrm{In}^{3+}$. The adjusted dipole moments of the first solvation shell water molecules for different ions are consistent with theory and $a b$ initio molecular dynamics (AIMD) simulations. In addition, we derived the physical relationship between the $C_{4}$ parameter and induced dipole moment, which has excellent agreement with the simulation results we got. The concept and strategy of the current study can be further applied to biological systems such as metalloproteins. Considering the present study used a similar scheme to the fluctuating charge model, we believe it can bridge the 12-6-4 model and the fluctuating charge model.

\section{COMPUTATIONAL METHODS}

The potential function of the 12-6 Lennard-Jones nonbonded model in the AMBER force field (Cornell et al., 1995) is shown below:

$$
\begin{aligned}
U_{i j}\left(r_{i j}\right) & =\frac{e^{2} Q_{i} Q_{j}}{r_{i j}}+\frac{C_{12}^{i j}}{r_{i j}^{12}}-\frac{C_{6}^{i j}}{r_{i j}^{6}} \\
& =\frac{e^{2} Q_{i} Q_{j}}{r_{i j}}+\varepsilon_{i j}\left[\left(\frac{R_{m i n, i j}}{r_{i j}}\right)^{12}-2\left(\frac{R_{m i n, i j}}{r_{i j}}\right)^{6}\right]
\end{aligned}
$$

The potential function of the 12-6-4 nonbonded model ( $\mathrm{Li}$ and Merz, 2014; Li et al., 2015a; Li et al., 2015b) is shown as follows:

$$
\begin{aligned}
U_{i j}\left(r_{i j}\right) & =\frac{e^{2} Q_{i} Q_{j}}{r_{i j}}+\frac{C_{12}^{i j}}{r_{i j}^{12}}-\frac{C_{6}^{i j}}{r_{i j}^{6}}-\frac{C_{4}^{i j}}{r_{i j}^{4}} \\
& =\frac{e^{2} Q_{i} Q_{j}}{r_{i j}}+\varepsilon_{i j}\left[\left(\frac{R_{m i n, i j}}{r_{i j}}\right)^{12}-2\left(\frac{R_{m i n, i j}}{r_{i j}}\right)^{6}\right]-\frac{C_{4}^{i j}}{r_{i j}^{4}}
\end{aligned}
$$

In these equations, $U_{i j}$ is the potential energy between atoms $i$ and $j . r_{i j}$ is the distance between the two atoms. $Q_{i}$ and $Q_{j}$ are the particle charges of atoms $i$ and $j$, respectively. $e$ is the charge of the proton. $C_{12}^{i j} / r_{i j}^{12}$ is the Pauli repulsive term, $-C_{6}^{i j} / r_{i j}^{6}$ is the dispersion attractive term, while $-C_{4}^{i j} / r_{i j}^{4}$ is the ion-induced dipole term. $R_{m i n, i j}$ is the distance between atoms $i$ and $j$ where the Lennard-Jones potential has its minimum, while $\varepsilon_{i j}$ is the well depth of the Lennard-Jones potential at this distance. The Berthelot-Lorentz (LB) combining rules are used in the AMBER force field: (Cornell et al., 1995):

$$
\begin{aligned}
R_{\text {min }, i j} & =\frac{R_{\text {min, }, i i}}{2}+\frac{R_{\text {min }, j j}}{2} \\
\varepsilon_{i j} & =\sqrt{\varepsilon_{i} \times \varepsilon_{j}}
\end{aligned}
$$

In the current study, the OPC3 water model was used to simulate the solvent as it represents an accuracy limit for the rigid three-point water models.(Izadi and Onufriev, 2016). The thermodynamic integration (TI) method (Kollman, 1993) was employed to calculate the $\Delta G$ values. Calculations of the $\Delta G$ values were performed under the NVT ensemble by assuming that the Gibbs free energy change is identical to the Helmholtz 
free energy change. The IOD and $\mathrm{CN}$ values were obtained based on the radial distribution function (RDF) between the metal ion and water oxygen atoms. Specific steps are described as follows.

For the 12-6 model with regular charges, we calculated the HFE, IOD, and $\mathrm{CN}$ values with the method described previously (Li et al., 2020, Li et al., 2021; Sengupta et al., 2021). Among them the regular MD simulations were used to obtain the IOD and CN values.

For the 12-6 model with updated charges, we calculate the HFE, IOD, and CN values as described below. By treating the last snapshot of the regular MD simulations mentioned above as initial structure, we performed a set of TI calculations to obtain $\Delta G_{1 \text {,ind }}$ for increasing the dipole moments of the six closest water molecules from the metal ion. Afterwards, we performed a set of TI calculations in the reverse manner to get $\Delta G_{2, \text { ind }}$ for decreasing the dipole moments of these water molecules to the original value. The final HFE was calculated as the sum of the HFE for the 12-6 model with regular charges and $\left(\Delta G_{1, \text { ind }}-\Delta G_{2, \text { ind }}\right) / 2$. These TI calculations were performed with linear scaling: $U(\lambda)=(1-\lambda) U_{0}+\lambda U_{1}$. With $U_{0}$ and $U_{1}$ represent the initial and final Hamiltonians, respectively. Seven $\lambda$ windows (with $\lambda=$ $0.02544,0.12923,0.29707,0.5,0.70292,0.87076$, and 0.97455 ) were used for each set of TI calculations, with each window had $100 \mathrm{ps}$ equilibration and $200 \mathrm{ps}$ production. After finishing the TI calculations, the Gaussian quadrature was used to calculate $\Delta G_{1, \text { ind }}$ and $\Delta G_{2, \text { ind }}: \Delta G=\sum_{i} w_{i}\langle\partial U / \partial \lambda\rangle_{i}$. Herein, $w_{i}$ is the weight for window $i$, while $\langle\partial U / \partial \lambda\rangle_{i}$ is the averaged $\partial U / \partial \lambda$ value for window $i$. Considering the uncertainties of the calculated HFEs are $\sim 1 \mathrm{kcal} / \mathrm{mol}$ for monovalent and divalent metal ions and $\sim 2 \mathrm{kcal} / \mathrm{mol}$ for the highly charged metal ions when using the 12-6 model with regular charges, (Li et al., 2013; Li et al., 2015a; Li et al., 2015b), we estimated the uncertainties of the final HFE values are $\sim 3 \mathrm{kcal} / \mathrm{mol}$ or less based on the uncertainty propagation, by assuming the additional TI calculations provide an uncertainty of similar magnitude.

For $\mathrm{Fe}^{3+}$ and $\mathrm{In}^{3+}$, a weak restraint was used for the additional TI calculations due to there was a CN switching: the $\mathrm{CN}$ value was eight or so when these water molecules have fixed charges of the OPC 3 water model, while it changes to six for the equilibrated structure of the system when the six closest water molecules to the ion have enlarged dipole moments. To prevent these six water molecules leaving the first solvation shell (due to they have "induced dipoles" which are caused by their proximity to the ion), when calculating $\Delta G_{1, \text { ind }}$ and $\Delta G_{2, \text { ind }}$, a one-side weak restraint of $U=k\left(r-r_{e q}\right)^{2}$ with $k$ equals $10 \mathrm{kcal} /\left(\mathrm{mol} \cdot \AA^{2}\right)$ was applied to each of these six ion-oxygen distances when $r$ is bigger than $2.5 \AA$. The same weak restraint was applied to the initial and final states during the TI calculations. Because the $\mathrm{CN}$ switching was involved, we carried out longer simulations with each $\lambda$ window had $200 \mathrm{ps}$ equilibration and $800 \mathrm{ps}$ production for the additional TI calculations of $\mathrm{Fe}^{3+}$ or $\mathrm{In}^{3+}$.

We noticed that there were free energy changes by applying the restraints mentioned above. To calculate these free energy changes (that is the free energy change between the systems with and without restraints for each of the system with regular charges and the system with updated charges), the free energy perturbation (Zwanzig, 1954) equation was used (with assuming the ensembles $\mathrm{A}$ and $\mathrm{B}$ are the same):

$$
\Delta G_{\mathrm{A} \rightarrow \mathrm{B}}=\langle G\rangle_{\mathrm{B}}-\langle G\rangle_{\mathrm{A}}=k_{\mathrm{B}} T \ln \left\langle e^{\left(E_{\mathrm{B}}-E_{\mathrm{A}}\right) / k_{\mathrm{B}} T}\right\rangle_{\mathrm{B}}
$$

Where A represents the system without the restraints, and B represents the system with the restraints. Based on Eq. 5, we obtained the $\Delta G_{\mathrm{A} \rightarrow \mathrm{B}}$ value for the system with regular charges (herein termed as $\Delta G_{\text {nores } \rightarrow \text { res }}^{\text {regular }}$ ), and the $\Delta G_{\mathrm{A} \rightarrow \mathrm{B}}$ value for the system with updated charges (termed as $\Delta G_{\text {nores } \rightarrow \text { res }}^{\text {updat }}$ ). For each of the system with regular charges and the system with updated charges, by starting from the same final structure of the regular MD simulations mentioned above, 2 ns NPT equilibration plus $2 \mathrm{~ns}$ NVT production were performed, with the one-side restraints (mentioned in the last paragraph) applied to both the equilibration and production. The six ion-oxygen distances were collected every $10 \mathrm{fs}$, and they were used to calculate the $E_{\mathrm{B}}-E_{\mathrm{A}}$ values afterwards, providing 200,000 data points for calculating each of $\Delta G_{\text {nores } \rightarrow \text { res }}^{\text {regular }}$ and $\Delta G_{\text {nores } \rightarrow \text { res }}^{\text {update }}$. Both these two $\Delta G$ values should be non-negative as it is always true that $E_{\mathrm{B}} \geq E_{\mathrm{A}}$. Our calculations showed that the result of $\Delta G_{\text {nores } \rightarrow \text { res }}^{\text {regularQ }}-\Delta G_{\text {nores } \rightarrow \text { res }}^{\text {updateQ }}$ is below $1 \mathrm{kcal} / \mathrm{mol}$ for $\mathrm{Fe}^{3+}$ or $\mathrm{In}^{3+}$, which is below the uncertainty of the calculated HFEs, so we did not account these two $\Delta G$ values in the final reported HFE values.

Moreover, also by starting from the final structure of the regular MD simulations, we increased the dipole moments of the six closest water molecules of the metal ion by adjusting their atomic charges, and then we performed $2 \mathrm{~ns}$ NPT equilibration with reassigned initial velocities. Then we carried out $2 \mathrm{~ns}$ NVT production simulation with the snapshots saved every 500 fs. No restraints were applied in these MD simulations. Afterwards, the RDF between the metal ion and water oxygen atoms was calculated based on the production trajectory. Finally, the IOD and CN values were calculated based on this RDF as we obtained the IOD and CN values for the system with regular charges.

A quasi-cubic water box with a side length of $40 \AA$ and periodic boundary conditions were used in our MD simulations (including the TI simulations). The particle mesh Ewald (PME) method (Darden et al., 1993; Essmann et al., 1995) was used to deal with the long-range electrostatic interactions. A cut-off of $10 \AA$ was used for the nonbonded interactions in the real space. The size of the charge grid was set to 48 in each of the three dimensions in the reciprocal space. A time-step of $1 \mathrm{fs}$ was used, and the three-point "SHAKE" algorithm (Miyamoto and Kollman, 1992) was employed to constrain the geometries of the water molecules with a tolerance of $10^{-5} \AA$ for each bond. The Langevin algorithm with a collision frequency of $2 \mathrm{ps}^{-1}$ was used to control the temperature. The Brenden's barostat with a relaxation time of $1 \mathrm{ps}$ was employed to control the pressure in the MD simulations in the NPT ensemble. All the simulations were performed using the pmemd. cuda (Salomon-Ferrer et al., 2013; Lee et al., 2020) program in the AMBER software package 
(Assisted Model Building with Energy Refinement (AMBER), RRID:SCR_014230, and AmberTools, RRID:SCR_018497) (Case et al., 2020).

\section{RESULTS AND DISCUSSION}

\section{Experimental Data}

The experimental HFE, IOD, and $\mathrm{CN}$ values for the $\mathrm{Mg}^{2+}$, $\mathrm{Zn}^{2+}, \mathrm{Al}^{3+}, \mathrm{Fe}^{3+}$, and $\mathrm{In}^{3+}$ ions are shown in Table 1. The Marcus HFE set (Marcus, 1991) was used is because it could be considered as a set of "intrinsic" HFEs, which only considers the free energy change caused by the ion-water interactions but not accounts the free energy change for ion crossing the gas-liquid interface. This is consistent with the free energy calculations performed in this study that used periodic boundary conditions, as discussed previously ( $\mathrm{Li}$ et al., 2020; Sengupta et al., 2021).

\section{CN Overestimation Issue of the 12-6-4 Model}

Previously, we have shown that when in conjunction with the OPC3 water model, the 12-6 model could not simultaneously reproduce the experimental HFE and IOD values for each of $\mathrm{Mg}^{2+}, \mathrm{Zn}^{2+}, \mathrm{Al}^{3+}, \mathrm{Fe}^{3+}$, and $\mathrm{In}^{3+}$ (Li et al., 2020; Li et al., 2021). Specifically, when the $12-6$ model was able to reproduce the experimental HFE, it underestimated the IOD by $0.11,0.22,0.52,0.48$, and $0.31 \AA$ for $\mathrm{Mg}^{2+}, \mathrm{Zn}^{2+}$, $\mathrm{Al}^{3+}, \mathrm{Fe}^{3+}$, and $\mathrm{In}^{3+}$, respectively ( $\mathrm{Li}$ et al., 2020; $\mathrm{Li}$ et al.,2021). When the 12-6 model was able to reproduce the experimental IOD, it provided the HFE less negative than the experimental value by $33,63,152,177$, and $126 \mathrm{kcal} / \mathrm{mol}$ for $\mathrm{Mg}^{2+}, \mathrm{Zn}^{2+}, \mathrm{Al}^{3+}, \mathrm{Fe}^{3+}$, and $\mathrm{In}^{3+}$, respectively (Li et al., 2020; Li et al., 2021). Moreover, we have also parameterized the 12-6-4 model for these ions, which showed significant improvement than the 12-6 model: the 12-6-4 model could simultaneously reproduce the experimental HFE and IOD values for each of these ions (Li et al., 2020; Li et al., 2021). However, the 12-6-4 model overestimated the $\mathrm{CN}$ values for some highly charged ions (Li et al., 2015a; Li et al., 2021). In Table 2 we showed the 12-6-4 parameters for $\mathrm{Mg}^{2+}, \mathrm{Zn}^{2+}$, $\mathrm{Al}^{3+}, \mathrm{Fe}^{3+}$, and $\mathrm{In}^{3+}$ in conjunction with the OPC3 water model, and the simulated HFE, IOD, and CN values from the previous studies (Li et al., 2020; Li et al., 2021). It can be seen

TABLE 1 | Experimental HFE, IOD, and $\mathrm{CN}$ values for $\mathrm{Mg}^{2+}, \mathrm{Zn}^{2+}, \mathrm{Al}^{3+}, \mathrm{Fe}^{3+}$, and $\ln ^{3+}$.

\begin{tabular}{lccc} 
& HFE $(\mathbf{k c a l} / \mathbf{m o l})^{\mathbf{a}}$ & IOD $(\AA)^{\boldsymbol{b}}$ & $\mathbf{C N}^{\boldsymbol{b}}$ \\
\hline $\mathrm{Mg}^{2+}$ & -437.4 & $2.09 \pm 0.04$ & 6 \\
$\mathrm{Zn}^{2+}$ & -467.3 & $2.09 \pm 0.06$ & 6 \\
$\mathrm{Al}^{3+}$ & $-1,081.5$ & $1.88 \pm 0.01$ & 6 \\
$\mathrm{Fe}^{3+}$ & $-1,019.4$ & $2.03 \pm 0.01$ & 6 \\
$\mathrm{In}^{3+}$ & -951.2 & 2.15 & 6
\end{tabular}

${ }^{a}$ From Marcus (1991).

${ }^{\text {b} F r o m ~ M a r c u s ~(1988) . ~}$ that the 12-6-4 model overestimated the $\mathrm{CN}$ values of $\mathrm{Fe}^{3+}$ and $\mathrm{In}^{3+}$ as 6.7 and 8 , which is significantly higher than the experimentally determined $\mathrm{CN}$ values of 6 (see Table 1 ). In addition, to better understand the influence of the $C_{4}$ term, we calculated the HFE, IOD, and $\mathrm{CN}$ values by ignoring the $\mathrm{C}_{4}$ term in the potential (i.e. using the regular 12-6 model with the same Lennard-Jones parameters) in the current study. These results are shown in Table 2 as well. We can see that when ignoring the $C_{4}$ term, the simulated HFE changed to less negative by $46,78,201,195$, and $139 \mathrm{kcal} /$ mol for $\mathrm{Mg}^{2+}, \mathrm{Zn}^{2+}, \mathrm{Al}^{3+}, \mathrm{Fe}^{3+}$, and $\mathrm{In}^{3+}$, respectively. Meanwhile, the simulated IOD increased by $0.04,0.06$, $0.07,0.13$, and $0.06 \AA$ for $\mathrm{Mg}^{2+}, \mathrm{Zn}^{2+}, \mathrm{Al}^{3+}, \mathrm{Fe}^{3+}$, and $\mathrm{In}^{3+}$, respectively. These values highlighted the importance of the $\mathrm{C}_{4}$ term. Moreover, the $\mathrm{CN}$ value of the $\mathrm{Fe}^{3+}$ ion increased from 6.7 to 8.1, showing an enlarged overestimation when ignoring the $C_{4}$ term.

\section{Solution of the CN Overestimation Issue}

The CN overestimation issue of the 12-6-4 model not only exists for $\mathrm{Fe}^{3+}$ and $\mathrm{In}^{3+}$ but also for other highly charged metal ions such as $\mathrm{Tl}^{3+}$ (Li et al., 2015a; Li et al., 2021). We have discussed that this artifact was caused by the fixed charge nature of the first solvation water molecules. As the ion charge increases, the first solvation shell water molecules should have larger induced dipole moments caused by the ion-induced dipole interaction, yielding a stronger repulsion between them. However, this behavior has not been considered by the current 12-6-4 scheme, which only takes into account the ion-induced dipole interaction between ion and water molecules but not considers its influence on the water-water interactions. Hence, to solve the $\mathrm{CN}$ overestimation issue, we increased the dipole moments of the first solvation shell water molecules explicitly to account for their enlarged dipole moments caused by the ion-induced dipole interaction. We understood that this strategy was not perfect as it did not consider the charge fluctuations along with each subtle configuration change inside the first solvation shell and did not change the water dipole moments in the second solvation shell or beyond. However, we believed that this strategy could well serve for a qualitative and demonstration purpose. For example, the $a b$ initio MD simulations indicated that the water molecules in the second solvation shell of $\mathrm{Zn}^{2+}, \mathrm{Al}^{3+}$, and $\mathrm{Fe}^{3+}$ had almost identical dipole moments as those of bulk water molecules (Bogatko et al., 2010; Cauët et al., 2010).

By adjusting the dipole moments of the water molecules in the first solvation shell (i.e., the six water molecules which are closest to the metal ion), we were able to reproduce the experimental HFEs within $1 \mathrm{kcal} / \mathrm{mol}$ by using the $12-6$ model. Using the same water charge parameters, we calculated the IOD and $\mathrm{CN}$ values as well. The parameters and simulated results are shown in Table 3. The LennardJones parameters of metal ions employed in these simulations are the same as described in Table 2. We can see that these parameters could well reproduce the experimental IOD and $\mathrm{CN}$ values with reasonable accuracy as well. Specifically, in order to reproduce the experimental HFEs, we need to increase 
TABLE 2 | The 12-6 and $\mathrm{C}_{4}$ parameters and the simulated HFE, IOD, and CN values for $\mathrm{Mg}^{2+}, \mathrm{Zn}^{2+}, \mathrm{Al}^{3+}, \mathrm{Fe}^{3+}$, and In ${ }^{3+}$ in conjunction with the OPC3 water model. ${ }^{\text {a }}$

\begin{tabular}{|c|c|c|c|c|c|c|}
\hline & $R_{\min } / 2(\AA)$ & $\varepsilon$ (kcal/mol) & $C_{4}\left(\mathrm{kcal} / \mathrm{mol} \cdot \AA^{4}\right)$ & HFE (kcal/mol) & IOD (Å) & $\mathrm{CN}$ \\
\hline $\mathrm{Mg}^{2+}$ & 1.433 & 0.02174524 & 117 & -438.0 & 2.10 & 6.0 \\
\hline $\mathrm{Mg}^{2+}$ & 1.433 & 0.02174524 & $\mathrm{~N} / \mathrm{A}$ & -392.0 & 2.14 & 6.0 \\
\hline $\mathrm{Zn}^{2+}$ & 1.441 & 0.02343735 & 199 & -467.3 & 2.09 & 6.0 \\
\hline $\mathrm{Zn}^{2+}$ & 1.441 & 0.02343735 & $\mathrm{~N} / \mathrm{A}$ & -389.8 & 2.15 & 6.0 \\
\hline $\mathrm{Al}^{3+}$ & 1.361 & 0.01031847 & 363 & -1081.3 & 1.88 & 6.0 \\
\hline $\mathrm{Al}^{3+}$ & 1.361 & 0.01031847 & $\mathrm{~N} / \mathrm{A}$ & -882.0 & 1.95 & 6.0 \\
\hline $\mathrm{Fe}^{3+}$ & 1.455 & 0.02662782 & 429 & -1019.0 & 2.03 & 6.7 \\
\hline $\mathrm{Fe}^{3+}$ & 1.455 & 0.02662782 & $\mathrm{~N} / \mathrm{A}$ & -824.3 & 2.16 & 8.1 \\
\hline $\ln ^{3+}$ & 1.487 & 0.03507938 & 330 & -951.4 & 2.16 & 8.0 \\
\hline $\ln ^{3+}$ & 1.487 & 0.03507938 & $\mathrm{~N} / \mathrm{A}$ & -812.0 & 2.22 & 8.0 \\
\hline
\end{tabular}

${ }^{a}$ The 12-6-4 parameters and simulated HFE, IOD, and $\mathrm{CN}$ values for $\mathrm{Mg}^{2+}$ and $\mathrm{Zn}^{2+}$ are from Ref. (Li et al., 2020). The 12-6-4 parameters and simulated HFE, IOD, and CN values for A ${ }^{3+}$, $\mathrm{Fe}^{3+}$, and $\mathrm{In}^{3+}$ are from Ref. (Li et al., 2021).

TABLE 3 | Simulated HFE, IOD, and CN values when using the 12-6 model with the adjusted charges for the six closest molecules to the metal ion.

\begin{tabular}{|c|c|c|c|c|c|c|c|c|c|}
\hline & $R_{\min } / 2(\AA ̊)$ & $\begin{array}{c}\varepsilon \\
\text { (kcal/mol) }\end{array}$ & $\begin{array}{l}Q_{0} \\
(e)^{a}\end{array}$ & $\begin{array}{l}Q_{\mathrm{H}} \\
(e)^{a}\end{array}$ & $\begin{array}{c}\mu_{\text {tot }} \\
\text { of Water } \\
(D)^{a}\end{array}$ & $\begin{array}{c}\mu_{\text {ind }} \\
\text { of Water } \\
(D)^{a}\end{array}$ & HFE (kcal/mol) & IOD (Å) & $\mathrm{CN}$ \\
\hline $\mathrm{Mg}^{2+}$ & 1.433 & 0.02174524 & -1.02 & +0.51 & 2.77 & 0.34 & -437.5 & 2.12 & 6.0 \\
\hline $\mathrm{Zn}^{2+}$ & 1.441 & 0.02343735 & -1.1096 & +0.5548 & 3.01 & 0.58 & -467.5 & 2.12 & 6.0 \\
\hline $\mathrm{Al}^{3+}$ & 1.361 & 0.01031847 & -1.194 & +0.597 & 3.24 & 0.81 & $-1,082.1$ & 1.92 & 6.0 \\
\hline $\mathrm{Fe}^{3+}$ & 1.455 & 0.02662782 & -1.222 & +0.611 & 3.32 & 0.89 & $-1,019.0$ & 2.02 & 6.0 \\
\hline $\ln ^{3+}$ & 1.487 & 0.03507938 & -1.152 & +0.576 & 3.13 & 0.70 & -951.9 & 2.07 & 6.0 \\
\hline
\end{tabular}

${ }^{a}$ For the OPC3 water model, the charge of the water oxygen atom is $-0.8952 \mathrm{e}$, the charge of the water hydrogen atom is +0.4476 e, and the dipole moment of the water molecule is $2.43 \mathrm{D}$ (Izadi and Onufriev, 2016).

the dipole moments of the first solvation shell water molecules by $0.34,0.58,0.81,0.89$, and $0.70 \mathrm{D}$ for $\mathrm{Mg}^{2+}, \mathrm{Zn}^{2+}, \mathrm{Al}^{3+}, \mathrm{Fe}^{3+}$, and $\mathrm{In}^{3+}$ respectively. This is consistent with the trend that ion with a higher charge will cause larger induced dipoles of the surrounding molecules, and $\mathrm{Zn}^{2+}$ has a stronger such ability than $\mathrm{Mg}^{2+}$ (by considering $\mathrm{Zn}^{2+}$ has the same IOD but a more negative HFE than that of $\mathrm{Mg}^{2+}$, see Table $\mathbf{1}$ ).

\section{Induced Dipole Moments Predicted by Theory and Quantum Calculations}

To better understand the ion-induced dipole interaction, we performed the following calculations. The induced dipole of a particle caused by a point charge can be calculated based on the following equation:

$$
\mu_{\text {ind }}=\alpha E=\alpha \frac{q}{4 \pi \varepsilon_{0} \varepsilon_{r} r^{2}}=\frac{\alpha_{0} q}{\varepsilon_{r} r^{2}}
$$

Where $\mu_{\text {ind }}$ is the induced dipole, $\alpha$ is the polarizability of the particle, $E$ is the electric field strength act on that particle, $q$ is the charge magnitude of the point charge, $r$ is the distance between the point charge and particle, $\varepsilon_{0}$ is the vacuum permittivity, $\varepsilon_{r}$ is the dielectric constant of the medium, $\alpha_{0}$ is the polarizability volume of the particle which equals $\alpha / 4 \pi \varepsilon_{0}$. The polarizability volume of a water molecule was determined to be $1.444 \AA^{3}$ experimentally (Eisenberg and Kauzmann, 1969). We can calculate its $\mu_{\text {ind }}$ when there is a monovalent ion placed $2.05 \AA$ away from it and $\varepsilon_{r}=1$ (as used in the AMBER force field (Cornell et al., 1995)) based on Eq. 6:

$$
\mu_{\text {ind }}=\frac{\alpha_{0} q}{\varepsilon_{r} r^{2}}=\frac{1.444 \times(+1 e)}{(2.05)^{2}} \AA \cdot e=1.65 \mathrm{D}
$$

The permanent dipole moment $\mu_{\text {per }}$ of a water molecule was determined to be $1.85 \mathrm{D}$ under gas phase (Dyke and Muenter, 1973). So this predicts that the total dipole moment $\mu_{\text {tot }}$ (i.e., $\mu_{\text {per }}+\mu_{\text {ind }}$ ) would be $3.5 \mathrm{D}$ for the water molecule inside a monovalent ion-water complex with the ion-oxygen distance as $2.05 \AA$, by assuming both $\mu_{\text {ind }}$ and $\mu_{\text {per }}$ point towards the monovalent ion. Because $\mu_{\text {ind }}$ is proportional to $q$, so we can get the $\mu_{\text {tot }}$ of the water molecule is 5.15 and $6.80 \mathrm{D}$ in the analogous divalent ion-water complex and trivalent ion-water complex, respectively.

The above theoretical calculations assumed the water molecule was a single-point particle. Although it could provide some insights about the magnitude of $\mu_{\text {ind }}$, this description was not accurate. To further evaluate $\mu_{\text {ind }}$ of the water molecule in an

TABLE $4 \mid \mu_{\text {tot }}$ and $\mu_{\text {ind }}$ calculated at the B3LYP/6-31G** and PBE0/6-31G $\mathrm{G}^{\star \star}$ (values before and after slashes, respectively) levels of theory when placing a point charge on the central axis of a water molecule with OPC3 geometry and $2.05 \AA$ away from the coordinating water oxygen.

\begin{tabular}{lcc} 
Point charge (e) & $\mu_{\text {tot }}(\mathbf{D})$ & $\mu_{\text {ind }}(\mathbf{D})$ \\
\hline 0 & $1.96 / 1.99$ & $0.00 / 0.00$ \\
+1 & $2.74 / 2.77$ & $0.78 / 0.78$ \\
+2 & $3.47 / 3.49$ & $1.51 / 1.50$ \\
+3 & $4.17 / 4.19$ & $2.21 / 2.20$
\end{tabular}


ion-water complex, we performed quantum calculations for the dipole moment of a water molecule when placing a point charge on the central axis of the water molecule. The quantum calculations were performed using the B3LYP density functional (Becke, 1988; Lee et al., 1988; Becke, 1993; Stephens et al., 1994) with the $6-31 G^{* *}$ basis set (Hehre et al., 1972; Hariharan and Pople, 1973). To keep consistent with the MD study, geometry of the OPC3 water model was used for the water molecule. These quantum chemistry calculations were performed using the Gaussian16 software (Gaussian, RRID:SCR_014897) (Frisch et al., 2019). Herein, we put the point charge $2.05 \AA$ away from the coordinating water oxygen atom. The calculated results are shown in Table 4 . We can see that when the point charge has a magnitude of $+1 e$, the induced dipole of the water molecule is $0.78 \mathrm{D}$ (with subtracting 1.96 from $2.74 \mathrm{D}$ ). This value is only about half of the value calculated by using Eq. 6. However, in terms of the correlation between $\mu_{\text {ind }}$ and $q$, we can see a clear trend which is consistent with Eq. 6 that $\mu_{\text {ind }}$ is proportional to $q$. Moreover, to test the dependence of the calculated values on density functionals, we also calculated these values by using the PBE0 density functional (Perdew et al., 1996; Perdew et al., 1997; Adamo and Barone, 1999) with the $6-31 G^{\star *}$ basis set. The results are shown in Table 4 as well. We can see these values are very close to what we obtained by using the B3LYP density functional.

Although having a quantum description of the water molecule, the above quantum calculations still use a pointcharge representation for the metal ion. Furthermore, we did a literature investigation about previous work of AIMD simulations for metal ions in aqueous phase. We found that the magnitudes of the dipole enlargements for the first solvation shell water molecules of $\mathrm{Fe}^{3+}$ agreed well with our parameters shown in Table 3. Specifically, previous AIMD simulations of the $\mathrm{Zn}^{2+}$-aqueous system indicated the dipole moments of first solvation shell water molecules are 0.7 D larger than those of those of bulk water molecules (Cauët et al., 2010). Other studies indicated that this value is $0.2 \mathrm{D}$ for the $\mathrm{Mg}^{2+}$ ion (Lightstone et al., 2001) and 1.0 $\mathrm{D}$ for the $\mathrm{Al}^{3+}$ ion (Bogatko et al., 2010). All of these values have excellent agreement with our parameters in Table 3. Previous AIMD simulations found that the first solvation shell water molecules of $\mathrm{Fe}^{3+}$ had dipoles of $0.5 \mathrm{D}$ higher than those of bulk water molecules, (Bogatko et al., 2010), which only qualitatively agreed with our value in Table 3. This may be due to the challenge of simulating the d-orbital effect by using the classical force field. In general, the strategy employed in the current study not only reproduced multiple physical properties simultaneously through a physically meaningful way but also showed well agreement with AIMD simulations.

\section{Relationship Between the $\boldsymbol{C}_{\mathbf{4}}$ and $\mu_{\text {ind }}$ Values}

To elucidate the relationship between the $C_{4}$ parameter and $\mu_{\text {ind }}$, we performed the following derivation. The ion-induced dipole interaction potential can be described by the following equation:
TABLE 5 | Calculated $\mu_{\text {ind }}$ and $\mu_{\text {tot }}$ for the first solvation shell water molecules of different metal ions based on Eq. 14.

\begin{tabular}{lcccc} 
& $\boldsymbol{C}_{\mathbf{4}}\left(\mathbf{k c a l} / \mathbf{m o l} \cdot \AA^{\mathbf{4}}\right)$ & $\boldsymbol{r}(\mathbf{A})^{\mathbf{a}}$ & $\mu_{\text {ind }}(\mathbf{D})$ & $\mu_{\text {tot }}(\mathbf{D})^{\mathbf{b}}$ \\
\hline $\mathrm{Mg}^{2+}$ & 117 & 2.09 & 0.39 & 2.82 \\
$\mathrm{Zn}^{2+}$ & 199 & 2.09 & 0.66 & 3.09 \\
$\mathrm{Al}^{3+}$ & 363 & 1.88 & 0.99 & 3.42 \\
$\mathrm{Fe}^{3+}$ & 429 & 2.03 & 1.00 & 3.43 \\
$\mathrm{In}^{3+}$ & 330 & 2.16 & 0.68 & 3.11 \\
\hline
\end{tabular}

${ }^{a}$ Referred to the $I O D$ values in Table 1.

${ }^{b}$ By summing $\mu_{\text {per }}$ of the OPC3 water model $(2.43 D)$ and $\mu_{\text {ind }}$.

$$
\begin{aligned}
U(r) & =-\int_{0}^{E} \mu_{\text {ind }} d E=-\int_{0}^{E} \alpha E d E=-\alpha \int_{0}^{E} E d E=-\frac{1}{2} \alpha E^{2} \\
& =-\frac{1}{2} \alpha\left(\frac{q}{4 \pi \varepsilon_{0} \varepsilon_{r} r^{2}}\right)^{2}=-\frac{1}{2} \frac{\alpha_{0} q^{2}}{4 \pi \varepsilon_{0} \varepsilon_{r}^{2} r^{4}}
\end{aligned}
$$

Herein, $U(r)$ represents the energy needed to bring a neutral species from infinite away where the electric field strength equals zero to a certain position with an electric field strength of $E$. Because the ion-induced dipole term equals $-C_{4} / r^{4}$ in the 12-6-4 model, we can get:

$$
C_{4}=\frac{1}{2} \frac{\alpha_{0} q^{2}}{4 \pi \varepsilon_{0} \varepsilon_{r}^{2}}
$$

Moreover, we can obtain the following relationship based on Eq. 6:

$$
\mu_{\text {ind }} \varepsilon_{r} r^{2}=\alpha_{0} q
$$

By combining Eqs 9 and 10, we can obtain:

$$
C_{4}=\frac{1}{2} \frac{\alpha_{0} q^{2}}{4 \pi \varepsilon_{0} \varepsilon_{r}^{2}}=\frac{1}{2} \frac{\mu_{\text {ind }} r^{2} q}{4 \pi \varepsilon_{0} \varepsilon_{r}}
$$

We denote:

$$
k_{e}=\frac{1}{4 \pi \varepsilon_{0} \varepsilon_{r}}
$$

Hence, we can obtain:

$$
\begin{aligned}
C_{4} & =\frac{1}{2} k_{e} \mu_{\text {ind }} r^{2} q \\
\mu_{\text {ind }} & =\frac{2 C_{4}}{k_{e} q r^{2}}
\end{aligned}
$$

$k_{\mathrm{e}}$ is a constant of $8.988 \times 10^{9} \mathrm{~J} \mathrm{~m} / \mathrm{C}^{2}$ when $\varepsilon_{r}=1$, so we can calculate $\mu_{\text {ind }}$ based on $C_{4}, q$ and $r$ for each of the metal ions investigated in the present study. The results are shown in Table 5. These values showed excellent agreement with the values in Table 3, validating the computational strategy employed in the present study.

\section{Implications to Metalloproteins}

Previously, we found that the 12-6 model could not reproduce the experimental HFE and IOD values simultaneously when metal ion has a charge of +2 or higher. We attributed this deficiency to the overlook of the ion-induced dipole interaction and developed 
the 12-6-4 model accordingly. Encouragingly, the 12-6-4 model could simultaneously reproduce the experimental HFE and IOD values for various metal ions ( $\mathrm{Li}$ et al., 2013; Li and Merz, 2014; Li et al., 2015a; Li et al., 2015b). Moreover, we also found that the 126-4 model could provide increased stability than the 12-6 model when simulating a metalloprotein system (Li et al., 2021). In addition, Sengupta et al. have shown that the regular 12-6 model could not well simulate the chelate effect between ethylenediamine and metal ions, while the 12-6-4 model was able to well model this effect after parameter optimization (Sengupta et al., 2018). Furthermore, Song et al. showed that the 12-6-4 model could well simulate metal ion binding to a protein active site after parameter refinement (Song et al., 2020). These results indicated that the 12-6-4 model can overcome the deficiency of the 12-6 model and provide an improved representation of the metal ion containing systems.

Recently, Macchiagodena et al. developed a 12-6 model for fourcoordinated zinc sites in metalloproteins (Macchiagodena et al., 2019). In this model, they refitted the atomic charges of the coordinating groups in the ligating residues. In order to reproduce the mean distances between $\mathrm{Zn}^{2+}$ and the ligating atoms, they adjusted their Lennard-Jones parameters. In their final parameters, the charges of the ligating sulfur atoms increased by $6-19 \%$ when comparing to the charge of the corresponding sulfur atom in the AMBER force field, while the charges of the ligating nitrogen atoms increased by $75-134 \%$ when comparing to charges of the corresponding nitrogen atoms in the AMBER force field. Their parameters successfully reproduced the experimentally determined mean distances between $\mathrm{Zn}^{2+}$ and the ligating atoms and provided $\mathrm{Zn}^{2+}$ binding affinities which correlated well with the experimentally determined dissociation constants. The strategy employed in their study is similar to that was used in the present work which considers the polarization effect by adjusting the atomic charges of the coordinating groups. And this strategy could improve the representation of the classical force field just as the 12-6-4 model did.

\section{CONCLUSION}

Our previous research found that the widely used 12-6 LennardJones nonbonded model for ions could not reproduce the experimental HFE and IOD values simultaneously when ion has a charge of +2 or higher (Li et al., 2013; Li et al., 2015a; Li et al., 2015b). We discussed that this deficiency arises from the overlook of the ion-induced dipole interaction, which is proportional to $1 / r^{4}$ where $r$ is the atomic distance ( $\mathrm{Li}$ and Merz, 2014). Through an $-C_{4} / r^{4}$ term which takes the ioninduced dipole interaction into account, we proposed and parameterized the 12-6-4 model for various ions (Li and Merz, 2014; Li et al., 2015a; Li et al., 2015b). Encouragingly, the 12-6-4 model could well reproduce the experimental HFE and IOD values at the same time (Li and Merz, 2014; Li et al., 2015a; Li et al., 2015b). However, we discovered a $\mathrm{CN}$ overestimation issue for some highly charged ions, and we discussed that this artifact arises from the fact that the current 12-6-4 model did not consider the increased repulsion among the first solvation water molecules of the metal ion (Li et al., 2015a; Li et al., 2021).

In the present study, we simulated multiple divalent and trivalent ions $\left(\mathrm{Mg}^{2+}, \mathrm{Zn}^{2+}, \mathrm{Al}^{3+}, \mathrm{Fe}^{3+}\right.$, and $\left.\mathrm{In}^{3+}\right)$ by using the 12-6 model with adjusting the atomic charges in the first solvation shell water molecules to consider the ion-induced dipole effect. We showed that this strategy could well reproduce the experimental HFE and IOD values simultaneously for these ions and solve the $\mathrm{CN}$ overestimation issue of the 12-6-4 model for $\mathrm{Fe}^{3+}$ and $\mathrm{In}^{3+}$. In addition, we found that the enlarged magnitudes of the dipole moments of the first solvation shell water molecules in our simulations agreed well with previous AIMD simulations, further validating the current strategy. Besides, we derived the relationship between the $C_{4}$ parameter and the induced dipole moment according to the physical principles. Moreover, the induced dipole moments calculated based on the $C_{4}$ parameters showed good agreement with our simulation results. Finally, we discussed that both the 12-6-4 scheme and the 12-6 scheme with adjusting atomic charges could simulate the polarization effect in metalloprotein systems. Due to the fluctuating charge model also uses the 12-6 scheme with adjusting the atomic charges, we believe the present study can serve as a bridge between the 12-6-4 model and the fluctuating charge model.

\section{DATA AVAILABILITY STATEMENT}

The original contributions presented in the study are included in the article/supplementary material, further inquiries can be directed to the corresponding author.

\section{AUTHOR CONTRIBUTIONS}

PL designed and performed the research; PL analyzed the data and wrote the paper.

\section{ACKNOWLEDGMENTS}

This work was funded by the start-up funds from Loyola University Chicago (to PL). PL thanks Tai-Sung Lee at Rutgers University for the helpful discussion. PL acknowledges the Extreme Science and Engineering Discovery Environment (XSEDE) (Towns et al., 2014) for the computational support. XSEDE is funded by the National Science Foundation (NSF) with Grant Number ACI-1548562. Specifically, this work used the computational resources on Comet and Expanse at the San Diego Supercomputer Center (Allocation Number TG-CHE200102). 


\section{REFERENCES}

Adamo, C., and Barone, V. (1999). Toward Reliable Density Functional Methods without Adjustable Parameters: The PBE0 Model. J. Chem. Phys. 110, 6158-6170. doi:10.1063/1.478522

Andreini, C., Bertini, I., and Rosato, A. (2004). A Hint to Search for Metalloproteins in Gene banks. Bioinformatics 20, 1373-1380. doi:10.1093/bioinformatics/bth095

Becke, A. D. (1988). Density-functional Exchange-Energy Approximation with Correct Asymptotic Behavior. Phys. Rev. A. 38, 3098-3100. doi:10.1103/physreva.38.3098

Becke, A. D. (1993). Density-functional Thermochemistry. III. The Role of Exact Exchange. J. Chem. Phys. 98, 5648-5652. doi:10.1063/1.464913

Bogatko, S. A., Bylaska, E. J., and Weare, J. H. (2010). First Principles Simulation of the Bonding, Vibrational, and Electronic Properties of the Hydration Shells of the High-Spin Fe3+ Ion in Aqueous Solutions. J. Phys. Chem. A. 114, 2189-2200. doi:10.1021/jp904967n

Cauët, E., Bogatko, S., Weare, J. H., Fulton, J. L., Schenter, G. K., and Bylaska, E. J. (2010). Structure and Dynamics of the Hydration Shells of the Zn2+ Ion Fromab Initiomolecular Dynamics and Combinedab Initioand Classical Molecular Dynamics Simulations. J. Chem. Phys. 132, 194502. doi:10.1063/1.3421542

Cornell, W. D., Cieplak, P., Bayly, C. I., Gould, I. R., Merz, K. M., Jr, Ferguson, D. M., et al. (1995). A Second Generation Force Field for the Simulation of Proteins, Nucleic Acids, and Organic Molecules. J. Am. Chem. Soc. 117, 5179-5197. doi:10.1021/ja00124a002

Case, D. A., Ben-Shalom, I. Y., Brozell, S. R., Cerutti, D. S., Cheatham, T. E., Cruzeiro, V. W. D., et al. (2020). AMBER 2020, University of California, Scan Francisco.

Darden, T., York, D., and Pedersen, L. (1993). Particle Mesh Ewald: AnN.Log(N) Method for Ewald Sums in Large Systems. J. Chem. Phys. 98, 10089-10092. doi:10.1063/1.464397

De Vivo, M., Masetti, M., Bottegoni, G., and Cavalli, A. (2016). Role of Molecular Dynamics and Related Methods in Drug Discovery. J. Med. Chem. 59, 4035-4061. doi:10.1021/acs.jmedchem.5b01684

Duan, Y., and Kollman, P. A. (1998). Pathways to a Protein Folding Intermediate Observed in a 1-microsecond Simulation in Aqueous Solution. Science 282, 740-744. doi:10.1126/science.282.5389.740

Duarte, F., Bauer, P., Barrozo, A., Amrein, B. A., Purg, M., Åqvist, J., et al. (2014). Force Field Independent Metal Parameters Using a Nonbonded Dummy Model. J. Phys. Chem. B 118, 4351-4362. doi:10.1021/jp501737x

Dyke, T. R., and Muenter, J. S. (1973). Electric Dipole Moments of Low J States of H2O and D2O. J. Chem. Phys. 59, 3125-3127. doi:10.1063/1.1680453

Eisenberg, D. S., and Kauzmann, W. (1969). The Structure and Properties of Water. New York: Oxford University Press.

Essmann, U., Perera, L., Berkowitz, M. L., Darden, T., Lee, H., and Pedersen, L. G. (1995). A Smooth Particle Mesh Ewald Method. J. Chem. Phys. 103, 8577-8593. doi:10.1063/1.470117

Hariharan, P. C., and Pople, J. A. (1973). The Influence of Polarization Functions on Molecular Orbital Hydrogenation Energies. Theoret. Chim. Acta 28, 213-222. doi:10.1007/bf00533485

Hehre, W. J., Ditchfield, R, and Pople, J. A. (1972). Self-consistent Molecular Orbital Methods. XII. Further Extensions of Gaussian-type Basis Sets for Use in Molecular Orbital Studies of Organic Molecules. J. Chem. Phys. 56, 2257-2261. doi:10.1063/1.1677527

Hollingsworth, S. A., and Dror, R. O. (2018). Molecular Dynamics Simulation for All. Neuron 99, 1129-1143. doi:10.1016/j.neuron.2018.08.011

Izadi, S., and Onufriev, A. V. (2016). Accuracy Limit of Rigid 3-point Water Models. J. Chem. Phys. 145, 074501.

Kepp, K. P. (2012). Bioinorganic Chemistry of Alzheimer's Disease. Chem. Rev. 112, 5193-5239. doi:10.1021/cr300009x

Kollman, P. (1993). Free Energy Calculations: Applications to Chemical and Biochemical Phenomena. Chem. Rev. 93, 2395-2417. doi:10.1021/cr00023a004

Lee, C., Yang, W., and Parr, R. G. (1988). Development of the Colle-Salvetti Correlation-Energy Formula into a Functional of the Electron Density. Phys. Rev. B 37, 785-789. doi:10.1103/physrevb.37.785

Lee, T.-S., Allen, B. K., Giese, T. J., Guo, Z., Li, P., Lin, C., et al. (2020). Alchemical Binding Free Energy Calculations in AMBER20: Advances and Best Practices for Drug Discovery. J. Chem. Inf. Model. 60, 5595-5623. doi:10.1021/acs.jcim.0c00613

Li, P., and Merz, K. M., Jr. (2014). Taking into Account the Ion-Induced Dipole Interaction in the Nonbonded Model of Ions. J. Chem. Theor. Comput. 10, 289-297. doi:10.1021/ct400751u

Li, P., and Merz, K. M. (2017). Metal Ion Modeling Using Classical Mechanics. Chem. Rev. 117, 1564-1686. doi:10.1021/acs.chemrev.6b00440
Li, P., Roberts, B. P., Chakravorty, D. K., and Merz, K. M., Jr. (2013). Rational Design of Particle Mesh Ewald Compatible Lennard-Jones Parameters for +2 Metal Cations in Explicit Solvent. J. Chem. Theor. Comput. 9, 2733-2748. doi:10.1021/ct400146w

Li, P., Song, L. F., and Merz, K. M., Jr. (2015a). Parameterization of Highly Charged Metal Ions Using the 12-6-4 LJ-type Nonbonded Model in Explicit Water. J. Phys. Chem. B 119, 883-895. doi:10.1021/jp505875v

Li, P., Song, L. F., and Merz, K. M., Jr. (2015b). Systematic Parameterization of Monovalent Ions Employing the Nonbonded Model. J. Chem. Theor. Comput. 11, 1645-1657. doi:10.1021/ct500918t

Li, Z., Song, L. F., Li, P., and Merz, K. M., Jr. (2021). Parametrization of Trivalent and Tetravalent Metal Ions for the OPC3, OPC, TIP3P-FB, and TIP4P-FB Water Models. J. Chem. Theor. Comput. 17, 2342-2354. doi:10.1021/acs.jctc.0c01320

Li, Z., Song, L. F., Li, P., and Merz, K. M., Jr. (2020). Systematic Parametrization of Divalent Metal Ions for the OPC3, OPC, TIP3P-FB, and TIP4P-FB Water Models. J. Chem. Theor. Comput. 16, 4429-4442. doi:10.1021/ acs.jctc.0c00194

Lightstone, F. C., Schwegler, E., Hood, R. Q., Gygi, F., and Galli, G. (2001). A First Principles Molecular Dynamics Simulation of the Hydrated Magnesium Ion. Chem. Phys. Lett. 343, 549-555. doi:10.1016/s0009-2614(01)00735-7

Lindorff-Larsen, K., Piana, S., Dror, R. O., and Shaw, D. E. (2011). How FastFolding Proteins Fold. Science 334, 517-520. doi:10.1126/science.1208351

Macchiagodena, M., Pagliai, M., Andreini, C., Rosato, A., and Procacci, P. (2019). Upgrading and Validation of the AMBER Force Field for Histidine and Cysteine Zinc(II)-Binding Residues in Sites with Four Protein Ligands. J. Chem. Inf. Model. 59, 3803-3816. doi:10.1021/acs.jcim.9b00407

Marcus, Y. (1988). Ionic Radii in Aqueous Solutions. Chem. Rev. 88, 1475-1498. doi:10.1021/cr00090a003

Marcus, Y. (1991). Thermodynamics of Solvation of Ions. Part 5.-Gibbs Free Energy of Hydration at 298.15 K. J. Chem. Soc. Faraday Trans. 87, 2995-2999. doi: $10.1039 / \mathrm{ft} 9918702995$

Miyamoto, S., and Kollman, P. A. (1992). SETTLE: an Analytical Version of the SHAKE and RATTLE Algorithm for Rigid Water Models. J. Comput. Chem. 13, 952-962. doi:10.1002/jcc.540130805

Frisch, M. J., Schlegel, G. W. T., H. B., Scuseria, G. E., Robb, M. A., Cheeseman J. R., Scalmani, G., et al. (2019). Gaussian 16, Revision C. 01. Gaussian, Inc., Wallingford CT).

Perdew, J. P., Burke, K., and Ernzerhof, M. (1996). Generalized Gradient Approximation Made Simple. Phys. Rev. Lett. 77, 3865-3868. doi:10.1103/physrevlett.77.3865

Perdew, J. P., Burke, K., and Ernzerhof, M. (1997). Generalized Gradient Approximation Made Simple [Phys. Rev. Lett. 77, 3865 (1996)]. Phys. Rev. Lett. 78, 1396. doi:10.1103/physrevlett.78.1396

Peters, M. B., Yang, Y., Wang, B., Füsti-Molnár, L., Weaver, M. N., and Merz, K. M., Jr. (2010). Structural Survey of Zinc-Containing Proteins and Development of the Zinc AMBER Force Field (ZAFF). J. Chem. Theor. Comput. 6, 2935-2947. doi:10.1021/ct1002626

Sakharov, D. V., and Lim, C. (2009). Force fields Including Charge Transfer and Local Polarization Effects: Application to Proteins Containing Multi/heavy Metal Ions. J. Comput. Chem. 30, 191-202. doi:10.1002/jcc.21048

Salomon-Ferrer, R., Götz, A. W., Poole, D., Le Grand, S., and Walker, R. C. (2013). Routine Microsecond Molecular Dynamics Simulations with AMBER on GPUs. 2. Explicit Solvent Particle Mesh Ewald. J. Chem. Theor. Comput. 9, 3878-3888. doi:10.1021/ct400314y

Sengupta, A., Li, Z., Song, L. F., Li, P., and Merz, K. M., Jr. (2021). Parameterization of Monovalent Ions for the OPC3, OPC, TIP3P-FB, and TIP4P-FB Water Models. J. Chem. Inf. Model. 61, 869-880. doi:10.1021/acs.jcim.0c01390

Sengupta, A., Seitz, A., and Merz, K. M. (2018). Simulating the Chelate Effect. J. Am. Chem. Soc. 140, 15166-15169. doi:10.1021/jacs.8b09371

Song, L. F., Sengupta, A., and Merz, K. M., Jr. (2020). Thermodynamics of Transition Metal Ion Binding to Proteins. J. Am. Chem. Soc. 142, 6365-6374. doi:10.1021/jacs.0c01329

Stephens, P. J., Devlin, F. J., Chabalowski, C. F., and Frisch, M. J. (1994). Ab Initio calculation of Vibrational Absorption and Circular Dichroism Spectra Using Density Functional Force fields. J. Phys. Chem. 98, 11623-11627. doi:10.1021/j100096a001

Thomson, A. J., and Gray, H. B. (1998). Bio-inorganic Chemistry. Curr. Opin. Chem. Biol. 2, 155-158. doi:10.1016/s1367-5931(98)80056-2

Towns, J., Cockerill, T., Dahan, M., Foster, I., Gaither, K., Grimshaw, A., et al. (2014). XSEDE. Accelerating Scientific Discovery. Comput. Sci. Eng. 16, 62-74. doi:10.1109/mcse.2014.80 
Waldron, K. J., and Robinson, N. J. (2009). How Do Bacterial Cells Ensure that Metalloproteins Get the Correct Metal?. Nat. Rev. Microbiol. 7, 25-35. doi:10.1038/nrmicro2057

Woodson, S. A. (2005). Metal Ions and RNA Folding: a Highly Charged Topic with a Dynamic Future. Curr. Opin. Chem. Biol. 9, 104-109. doi:10.1016/j.cbpa.2005.02.004

Zhang, J., Yang, W., Piquemal, J.-P., and Ren, P. (2012). Modeling Structural Coordination and Ligand Binding in Zinc Proteins with a Polarizable Potential. J. Chem. Theor. Comput. 8, 1314-1324. doi:10.1021/ct200812y

Zwanzig, R. W. (1954). High-Temperature Equation of State by a Perturbation Method. I. Nonpolar Gases. J. Chem. Phys. 22, 1420-1426. doi:10.1063/1.1740409
Conflict of Interest: The author declares that the research was conducted in the absence of any commercial or financial relationships that could be construed as a potential conflict of interest.

Copyright $\odot 2021 \mathrm{Li}$. This is an open-access article distributed under the terms of the Creative Commons Attribution License (CC BY). The use, distribution or reproduction in other forums is permitted, provided the original author(s) and the copyright owner(s) are credited and that the original publication in this journal is cited, in accordance with accepted academic practice. No use, distribution or reproduction is permitted which does not comply with these terms. 